\title{
MORTALIDADE NEONATAL: CARACTERÍSTICAS ASSISTENCIAIS E BIOLÓGICAS DOS RECÉM-NASCIDOS E DE SUAS MÃES*
}

\author{
Mayrene Dias de Sousa Moreira1, Maria Aparecida Munhoz Gaíva², Rossana Marchese Bittencourt
}

\begin{abstract}
RESUMO: Estudo transversal-descritivo com abordagem quantitativa cujo objetivo foi analisar as características assistenciais e biológicas dos nascidos vivos que evoluíram para óbito no período neonatal no município de Cuiabá-Mato Grosso, no ano de 2010. Os dados foram obtidos das Declarações de Nascidos Vivos, Declarações de Óbito e prontuários hospitalares de fevereiro a abril de 2011, organizados no programa Excel 2007 e submetidos à análise estatística descritiva. Ocorreram 77 óbitos neonatais sendo que $72,8 \%$ na primeira semana de vida, $71,5 \%$ dos bebês tinham peso menor que 2.500 gramas ao nascer, $75,3 \%$ nasceram com menos de 38 semanas de gestação e $51,9 \%$ dos partos foram cesáreo. A maioria dos neonatos recebeu assistência durante o parto; entretanto, a maior parte deles teve algum grau de asfixia ao nascer e necessitou de reanimação cardiopulmonar. O conhecimento dos fatores relacionados à mortalidade neonatal pode contribuir para a sua prevenção.
\end{abstract}

PALAVRAS-CHAVE: Mortalidade neonatal; Recém-nascido; Fatores de risco.

\section{NEONATAL MORTALITY: BIOLOGICAL AND CARE CHARACTERISTICS OF THE NEW-BORNS AND THEIR MOTHERS}

ABSTRACT: A transversal-descriptive study with a quantitative approach, with the objective of analyzing the biologicaland care characteristics of live new-borns who died in the post-partum period in the town of Cuiabá in the state of Mato Grosso in 2010. The data was obtained form Certificates of Live Birth, Death Certificates and hospital notes from February to April 2011, organized in Excel 2007 and submitted to analysis via descriptive statistics. There were 77 neonatal deaths, of which $72.8 \%$ took place in the first week of life. $71.5 \%$ of the babies had a birth weight of below $2,500 \mathrm{~g}$, and $75.3 \%$ were born at less than 38 weeks. $51.9 \%$ of the births were caesarean. The majority of the newborns received care during birth; however, most had some degree of asphyxia on being born and needed cardiopulmonary resuscitation. Knowledge of the factors related to neonatal mortality can contribute to its prevention.

KEYWORDS: Neonatal mortality; New-born; Risk factors.

\section{MORTALIDAD NEONATAL: CARACTERÍSTICAS ASISTENCIALES Y BIOLÓGICAS DE LOS RECIÉN NACIDOS Y DE SUS MADRES}

RESUMEN: Estudio transversal descriptivo con abordaje cuantitativo cuyo objetivo fue analizar las características asistenciales y biológicas de los nacidos vivos que evolucionaron para óbito en el periodo neonatal en el municipio de Cuiabá - Mato Grosso, en el año de 2010. Los datos fueron obtenidos de las Declaraciones de Nacidos Vivos, Declaraciones de Óbito y prontuarios hospitalares de febrero a abril de 2011, organizados en el programa Excel 2007 y sometidos al análisis estadístico descriptivo. Ocurrieron 77 óbitos neonatales siendo $72,8 \%$ en la primera semana de vida, $71,5 \%$ de los bebés tenían peso menor que 2.500 gramas al nacer, $75,3 \%$ naceran con menos de 38 semanas de gestación y 51,9\% de los partos fueron cesáreo. La mayoria de los neonatos tuvo asistencia durante el parto; sin embargo, la mayor parte de ellos tuvo algun grado de asfixia al nacer y necesitó de reanimación cardiopulmonar. El conocimiento de los factores relacionados a la mortalidad neonatal puede contribuir para su prevención.

PALABRAS-CLAVE: Mortalidad neonatal; Recién nacido; Factores de riesgo.

*Este estudo faz parte do relatório de Iniciação Cientifica financiado pela Fundação de Amparo à Pesquisa do Estado de Mato Grosso, 2011.
${ }^{1}$ Graduanda em Enfermagem pela Faculdade de Enfermagem da Universidade Federal de Mato Grosso- UFMT. Aluna de Iniciação
Científica e Bolsista da Fundação de Amparo à Pesquisa do Estado de Mato Grosso.
${ }^{2}$ Enfermeira. Doutora em Enfermagem. Professora do Programa de Pós-Graduação da Faculdade de Enfermagem da UFMT. Líder do
Grupo de Pesquisa Projeto Argos. Pesquisadora do CNPq. Orientadora.
${ }^{3}$ Enfermeira. Mestranda pelo Programa de Pós-Graduação em Enfermagem da UFMT. Membro do Grupo de Pesquisa Projeto Argos.

Autor correspondente:

Recebido: 29/11/2011

Mayrene Dias de Sousa Moreira

Aprovado: 29/02/2012

Universidade Federal de Mato Grosso

Rua Desembargador José de Mesquita, 649-78005-560-Cuiabá-MT-Brasil

E-mail: mayrenemay@hotmail.com 


\section{INTRODUÇÃO}

Os coeficientes de mortalidade neonatal são considerados, atualmente, um dos principais indicadores para a avaliação dos serviços de saúde, sobretudo para a qualidade da assistência à gestante no pré-natal, parto, puerpério e cuidado neonatal ${ }^{(1-2)}$.

Os óbitos neonatais são considerados potencialmente evitáveis, desde que os serviços de saúde incorporem em sua rotina de trabalho a análise das características de sua ocorrência ${ }^{(1)}$. Conhecer essas características contribui para proteger e melhorar a saúde materno-infantil permitindo a identificação das dificuldades no acesso à assistência pré-natal, ao parto e aos cuidados ao neonato ${ }^{(3)}$. Além disso, a identificação dessas características permite reformular as práticas de saúde, tornando a assistência mais adequada e de qualidade.

Dentre as características biológicas da mortalidade neonatal, o baixo peso ao nascer e o nascimento pré-termo são considerados como fatores de risco ${ }^{(4-5)}$. Observa-se ainda, que há um risco maior de morte para o recémnascido se essas duas variáveis estiverem associadas ${ }^{(3)}$.

Em relação às características assistenciais podemos citar o número de consulta pré-natal e Apgar no $1^{\circ}$ e $5^{\circ}$ minuto como fatores de risco e por isso determinantes para a mortalidade neonatal ${ }^{(3,5)}$. Garantido à gestante um acompanhamento pré-natal de qualidade, com número adequado de consultas, assistência apropriada durante o parto, ao recém-nascido e a puérpera poderíamos ter uma redução na mortalidade neonatal ${ }^{(4,6-7)}$.

A mortalidade infantil vem reduzindo-se nos últimos anos no Brasil, porém o componente neonatal deste indicador continua elevado, tornando-se assim um importante empecilho para a efetiva redução da mortalidade infantil e de seus principais componentes ${ }^{(8)}$. No Brasil, em 2008, a mortalidade neonatal foi de 10,24/1.000 nascidos vivos (nv) sendo que na Região Centro-Oeste foi de 9,72/1.000 nv e no Estado de Mato Grosso 10,64/1.000 nv ${ }^{(9)}$. No Município de Cuiabá a mortalidade neonatal também é maior que a pós-neonatal, o que contribui para o índice elevado da mortalidade infantil (17,07/1.000 nv em 2008). Em 2008 o município teve aproximadamente 10,64 óbitos por $1.000 \mathrm{nv}$ no período neonatal, sendo 7,58 óbitos no período neonatal precoce e 3,05 óbitos no período neonatal tardio e 6,42 óbitos no período pós-neonatal ${ }^{(10)}$.

Considerando as altas taxas de mortalidade neonatal do Município de Cuiabá, a importância que este coeficiente tem no índice de mortalidade infantil e a sua relevância como indicador de saúde para a ava- liação e reformulação de políticas de saúde voltadas para a melhoria da qualidade da assistência de saúde à gestação (pré-natal), parto, pós- parto e ao período neonatal, justifica-se a realização de pesquisas sobre esta temática, especialmente aquelas que abordam as características da mortalidade neonatal, como se propõe neste estudo.

A partir do exposto, destaca-se a relevância desta pesquisa que teve como objetivo analisar as características assistenciais e biológicas dos nascidos vivos que evoluíram para óbito no município de Cuiabá-MT, no ano de 2010, e de suas mães.

\section{MÉTODO}

Trata-se de um estudo transversal, descritivo de abordagem quantitativa, que teve como população todos os nascidos vivos residentes em Cuiabá, Mato Grosso, que nasceram em 2010 e evoluíram para o óbito nesse mesmo ano, cujas mães residiam no município.

Para a coleta dos dados foi utilizado um questionário padronizado construído especialmente para o estudo. Os dados foram coletados nos meses de fevereiro a abril de 2011. Iniciou-se a coleta a partir da identificação de todos os óbitos dos nascidos vivos em Cuiabá no ano de 2010, através das Declarações de Óbitos (DO). Após essa identificação, coletaram-se os dados dos DO (variáveis: tipo de parto, peso ao nascer, idade no óbito, idade materna, duração da gestação, partos anteriores, número de filhos vivos e tipo de gestação) e das Declarações de Nascidos Vivos (DNV) (variáveis: Apgar no $1^{\circ}$ e $5^{\circ}$ minuto e número de consultas pré-natal).

Através dessas fontes de dados identificou-se o hospital de ocorrência dos nascimentos e óbitos e coletaram-se as informações que poderiam ter influência no óbito nos prontuários dos recém-nascidos (variáveis: reanimação cardíaca, uso de surfactante, uso de corticóide antenatal, profissional que realizou parto, tempo de bolsa rota e se cesárea qual a indicação). Os dados coletados foram arquivados no programa Excel versão 2007 e realizado a análise descritiva das variáveis do estudo.

Este estudo faz parte do projeto intitulado "Análise dos determinantes da mortalidade neonatal em CuiabáMT, 2010", vinculado ao Programa de Pós-Graduação, nível Mestrado, da Faculdade de Enfermagem da Universidade Federal de Mato Grosso, UFMT, que foi aprovado pelo Comitê de Ética em Pesquisa do Hospital Júlio Müller sob n. 968/CEP-HUJM/2010, conforme as diretrizes da Resolução 196/96 do Conselho Nacional de Saúde. 


\section{RESULTADOS}

No ano de 2010 ocorreram 77 óbitos neonatais no município de Cuiabá-MT, a Taxa de Mortalidade Neonatal (TMN) foi de 8,4 óbitos/1.000 nv, correspondendo a $63,1 \%$ dos óbitos infantis. A TMN Precoce foi de 6,10 óbitos/1.000 e a TMN tardia foi de 2,28 óbitos/1.000 nv.

A distribuição dos óbitos foram: $72,8 \%(56)$ ocorreram no período neonatal precoce e destes, $28,6 \%(22)$ ocorreram antes de 24 horas de nascido. O período neonatal tardio abrangeu 27,2\%(21) do total dos óbitos, e dentre estes, $11,7 \%(9)$ tinham entre 8 a 14 dias, $10,4 \%(8)$ de 15 a 21 dias, e 5,2\%(4) de 22 a 27 dias de nascimento.

Na tabela 1 são apresentados os óbitos segundo as variáveis: peso de nascimento, Apgar e a idade gestacional, com o valor total e o respectivo percentual de cada uma delas.

Dos recém-nascidos estudados, $84,4 \%$ (65) foram submetidos a reanimação cardiopulmonar, 10,4\%(8) não realizaram e 5,2\%(4) dos prontuários não havia registro. Neste estudo 51,9\%(40) dos recém-nascidos fizeram uso de surfactante, $44,9 \%$ 33) não fizeram uso e 5,2\%(4) estavam sem registro. Além disso, em 55,8\%(43) dos neonatos não foi administrado corticóide antenatal, 27,3\%(21) foi administrado e $16,9 \%$ (13) estavam sem registro.

Na Tabela 2 está apresentada a distribuição percentual dos óbitos segundo: a idade materna, os partos anteriores, tipo de parto enúmero de consultas de pré-natal. Em relação ao número de filhos vivos 59,7\%(46) das mães tinham zero filhos vivos, $29,9 \%(23)$ tinham de 1 a 2 filhos vivos, 10,4\%(8) tinham de 3 a 4 filhos vivos. Quanto ao tipo de gestação, 90,9\%(70) das gestações foram únicas e 9,1\%(7) foram duplas.

Ainda no que diz respeito às características assistenciais maternas, $92,2 \%(71)$ dos partos foram realizados pelo médico obstetra, 2,6\%(2) por enfermeira obstetra e médico obstetra, 1,3\%(1) médico não obste-

Tabela 1 - Características biológicas dos recém-nascidos que vieram a óbito em Cuiabá em 2010. Cuiabá, 2011

\begin{tabular}{|c|c|c|}
\hline Peso ao nascer & $\mathbf{N}$ & $\%$ \\
\hline Menor que $500 \mathrm{~g}$ & 5 & 6,5 \\
\hline 500 a $999 \mathrm{~g}$ & 25 & 32,5 \\
\hline 1000 a $1499 g$ & 10 & 13,0 \\
\hline 1500 a $1999 g$ & 8 & 10,4 \\
\hline 2000 a $2499 g$ & 7 & 9,1 \\
\hline 2500 a $2999 g$ & 9 & 11,7 \\
\hline 3000 a $3499 g$ & 9 & 11,7 \\
\hline 3500 a $3999 s$ & 3 & 3,9 \\
\hline Maior que $4000 \mathrm{~g}$ & 1 & 1,3 \\
\hline Total & 77 & 100 \\
\hline Apagar no $1^{\circ}$ minuto & $\mathbf{N}$ & $\%$ \\
\hline Menor que 7 & 46 & 59,7 \\
\hline Maior que 7 & 27 & 35,1 \\
\hline Sem registro & 4 & 5,2 \\
\hline Total & 77 & 100 \\
\hline Apagar no $5^{\circ}$ minuto & $\mathbf{N}$ & $\%$ \\
\hline Menor que 7 & 30 & 39,0 \\
\hline Maior que 7 & 43 & 55,8 \\
\hline Sem registro & 4 & 5,2 \\
\hline Total & 77 & 100 \\
\hline Idade gestacional & $\mathbf{N}$ & $\%$ \\
\hline 22 a 30 semanas & 41 & 53,2 \\
\hline 31 a 34 semanas & 8 & 10,4 \\
\hline 35 a 37 semanas & 9 & 11,7 \\
\hline 38 a 41 semanas & 18 & 23,4 \\
\hline Maior que 42 semanas & 1 & 1,3 \\
\hline Total & 77 & 100 \\
\hline
\end{tabular}


Tabela 2 - Características biológicas e assistenciais das mães dos recém-nascidos que vieram a óbito em Cuiabá em 2010. Cuiabá, 2011

\begin{tabular}{lcc}
\hline Idade materna & $\mathbf{N}$ & $\mathbf{\%}$ \\
\hline 10 a 14 anos & 2 & 2.6 \\
15 a 19 anos & 21 & 27,3 \\
20 a 24 anos & 21 & 27,3 \\
25 a 29 anos & 17 & 22,1 \\
30 a 34 anos & 11 & 14,3 \\
Maior que 35 anos & 5 & 6,5 \\
Total & 77 & 100 \\
\hline Partos anteriores & $\mathbf{N}$ & $\mathbf{\%}$ \\
\hline Prímiparas & 10 & 13 \\
Multíparas & 67 & 87 \\
Total & 77 & 100 \\
\hline Tipo de Parto & $\mathbf{N}$ & $\mathbf{\%}$ \\
Cesárea & 40 & 51,9 \\
Vaginal & 37 & 48,1 \\
Total & 77 & 100 \\
\hline Número de Consultas pré-natal & $\mathbf{N}$ & $\mathbf{\%}$ \\
\hline Ignorado & 3 & 3,9 \\
Nenhum & 4 & 5,2 \\
1 a 3 & 9 & 11,7 \\
4 a 6 & 36 & 46,8 \\
7 ou mais & 25 & 32,5 \\
Total & 77 & 100 \\
\hline
\end{tabular}

tra, 2,6\%(2) dos partos não tiveram assistência médica no período expulsivo pois ocorreram no domicílio e $1,3 \%(1)$ estava sem registro.

Dentre as indicações de partos cesarianos na população estudada destacaram-se amniorrexe prematura e sofrimento fetal agudo. Sobre o tempo de bolsa rota, $15,6 \%(12)$ dos partos tiveram tempo de ruptura da bolsa menor que 24 horas, em 13\%(10) o tempo foi maior que 24 horas, 49,4\%(38) apresentavam a bolsa íntegra e $22 \%(17)$ estavam sem registro.

\section{DISCUSSÃO}

O período neonatal precoce continua sendo uma variável de grande representatividade na mortalidade neonatal em Cuiabá, já que a primeira semana de vida foi o período de maior ocorrência de óbitos $(72,8 \%)$. Destacaram-se também as primeiras 24 horas de vida, com $28,6 \%$ dos óbitos. Outros estudos realizados no país sobre mortalidade neonatal reafirmam esses resultados, mostrando que aproximadamente $60 \%$ das mortes neonatais ocorrem neste período, sendo a maior proporção antes de os neonatos completarem um dia de $\operatorname{vida}^{(5-6)}$.

Sabendo da maior vulnerabilidade dos bebês no período neonatal precoce é imprescindível que seja oferecida uma assistência de qualidade neste momento, com equipamentos disponíveis, medicações e uma equipe multiprofissional preparada para atender ao recém-nascido, principalmente pediatras. Somente com toda essa atenção torna-se possível preservar a vida dessas crianças.

A maior proporção de neonatos de baixo peso, ou seja, peso de nascimento menor que $2.500 \mathrm{~g}$, encontrada na população estudada, comprova que o baixo peso ao nascer é um fator de risco para a mortalidade neona$\operatorname{tal}^{(7)}$, e à medida que o peso de nascimento aumenta, o risco de morte diminui significativamente, podendo então ser considerado como um fator determinante da sobrevivência infantil ${ }^{(11)}$.

Pode-se observar que a grande maioria dos neonatos eram prematuros (idade gestacional menor que 37 semanas). Há uma relação inversa entre idade gestacional e mortalidade neonatal, a probabilidade de morte neonatal diminui significativamente à medida que aumenta a duração da gestação ${ }^{(4,7)}$. 
O escore baixo do Apgar é um dos fatores de risco descritos na literatura que guardam maior associação com a morte neonatal, o que também se mostrou em Cuiabá. Estudos que analisaram os fatores associados com a mortalidade neonatal mostraram que os nascidos vivos com escore de Apgar inferior a 7 no quinto minuto de vida apresentaram uma chance maior de morte do que os nascidos com escores superiores a sete ${ }^{(3,12)}$. O índice de Apgar apresenta-se como um indicador para a mortalidade neonatal, portanto, a organização da atenção obstétrica e neonatal deve proporcionar a diminuição dos fatores que podem levar à hipóxia perinatal e, consequentemente, ao óbito neonatal ${ }^{(13)}$.

Neste estudo verificou-se que mais de $80 \%$ dos recémnascidos foram submetidos à reanimação cardiopulmonar. Este procedimento quando realizado na sala de parto é utilizada para prevenção de asfixia perinatal e aspiração de mecônio, dentre outros. Eé considerado uma importante ferramenta para a redução da mortalidade neonatal ${ }^{(7)}$.

O surfactante é uma substância fundamental na mecânica pulmonar, e sua deficiência causa a síndrome do desconforto respiratório do recém-nascido. Dentre os efeitos benéficos do uso do surfactante, os mais marcantes são a redução da mortalidade e da ocorrência de escape de ar (pneumotórax e enfisema intersticial pulmonar) ${ }^{(14)}$, já o corticóide antenatal é usado como profilaxia dos problemas respiratórios; é um procedimento de fácil utilização e um dos fatores de proteção contra a mortalidade neonatal ${ }^{(12)}$. Segundo estudo que analisou os determinantes da mortalidade neonatal de uma coorte de nascidos vivos, o uso de corticoesteróide antenatal foi um fator que se associou com a diminuição do risco de óbito neonatal. Este procedimento, utilizado como profilaxia do problema respiratório, é menos dispendioso, de mais fácil utilização e apresenta melhores resultados se comparado a outros ${ }^{(12)}$. A regionalização da assistência perinatal e o desenvolvimento das tecnologias para a assistência neonatal, como a terapia intensiva e o uso de corticóide e surfactante nos casos de prematuridade têm sido considerados elementos importantes na redução da mortalidade perinatal em diversos países ${ }^{(15)}$.

Neste estudo, uma parcela importante das mães era composta de jovens. A idade da mãe é um fator importante para avaliação dos riscos de mortalidade infantil e perdas fetais, os maiores riscos ocorrem para mães com idade muito jovens ou com idades avançadas ${ }^{(16)}$. Com relação à assistência ambulatorial materna, o número de consultas pré-natal foi um resultado preocupante na população estudada se considerarmos que o Ministério da Saúde define como adequado a realização de no mí- nimo seis consultas durante a gestação ${ }^{(17)}$. A partir dos resultados encontrados, pode-se questionar a qualidade dessas consultas, já que é através do acompanhamento pré-natal que se identifica os riscos e patologias que, quando tratadas oportunamente, diminuem a vulnerabilidade dos recém-nascidos ao óbito. A preocupação com a assistência pré-natal não deve ater-se somente a frequência às consultas, mas deve-se considerar também a qualidade da atenção, incluindo treinamento da equipe, instituição de protocolos clínicos, utilização correta de critérios de gestação de risco e a garantia de referência quando identificado alto risco ${ }^{(7)}$.

A Organização Mundial da Saúde considera adequado um percentual de até $15 \%$ de partos cesáreos, pois valores maiores ou muito menores do que este podem trazer riscos tanto para a mãe como para o concepto. O parto cesariano deve ser realizado somente quando há perigo para mãe ou para o bebê, pois como qualquer procedimento cirúrgico tem seus riscos. Além disso, o nascimento prematuro pode estar associado a partos operatórios desnecessários, realizados antes da hora ${ }^{(18)}$. A grande quantidade de partos cesáreos realizados nos óbitos analisados pode significar um diagnóstico precoce de situações de risco e, consequentemente, intervenção adequada, como pode ser também uma interrupção precoce da gravidez com o objetivo de realizar cesarianas agendadas ${ }^{(19)}$.

\section{CONCLUSÕES}

Este estudo permitiu descrever as principais características assistenciais e biológicas das mães e dos recém-nascidos, residentes em Cuiabá, que evoluíram para óbito no município em 2010. Essas características são consideradas como fatores de risco para o óbito neonatal.

Conclui-se que os óbitos foram precoces, em recém-nascidos de baixo peso e prematuros, com prevalência de algum grau de asfixia ao nascer, necessitando de reanimação cardiopulmonar. As mães foram caracterizadas como jovens e realizaram menos consultas pré-natal do que o recomendado e o parto foi na maioria cesáreo, fatores que podem contribuir para complicações e óbito.

Recomenda-se a realização de estudos que investiguem a associação multivariada dos fatores relacionados à mortalidade neonatal, pois através do conhecimento desses torna-se possível a avaliação e a reformulação de políticas de saúde voltadas para a melhoria da qualidade da assistência de saúde à gestação (pré-natal), ao parto, pós-parto e ao período neonatal. 


\section{REFERÊNCIAS}

1. Ministério da Saúde (BR). Manual de Vigilância do óbito infantil e fetal e do Comitê de prevenção do óbito Infantil e fetal. Brasília. [Internet] 2009. [acesso em 30 set 2009]. Disponível: http://portal.saude.gov.br/portal/ arquivos/pdf/Manual_Infantil_Fetal.pdf

2. Barreto JOM, Nery IS, Mendes YMMB. Mortalidade perinatal: uma análise com enfoque na evitabitabilidade. Cogitare enferm. 2011;16(1):88-95.

3. Fréu CM, Mottin LM, Migotti MD, Martinelli IB, Nunes ML, Geib LTC. Determinantes da mortalidade neonatal de uma coorte de nascidos vivos em Passo Fundo - RS, 2003-2004. Revista da AMRIGS. 2008;2(52):97-102.

4. Ortiz LP, Oushiro DA. Perfil da mortalidade neonatal no Estado de São Paulo. São Paulo em Perspec. 2008;22(1):19-29.

5. Maran E, Uchimura TT. Mortalidade Neonatal: fatores de risco em um município no sul do Brasil. Rev. eletron. enfermagem. [Internet] 2008;10(1) [acesso em 29 set 2010]. Disponível: http://www.fen.ufg.br/revista/v10/ n1/v10n1a03.htm

6. Lima EFA, Sousa AI, Primo CC. Mortalidade Neonatal em Serra, Espírito Santo, 2001-2005. Rev enferm UERJ. 2008;16(2):162-7.

7. Ferrari LSL, Brito ASJ, Carvalho ABR, Gonzáles MRC. Mortalidade neonatal no Município de Londrina, Paraná, Brasil, nos anos 1994, 1999 e 2002. Cad. saude publica. 2006;22(5):1063-71.

8. Organização Pan-Americana da Saúde. Informe de Situação e Tendências: demografia e saúde/ Rede Interagencial de Informações para Saúde. Brasília. [Internet] 2009. [acesso em 20 nov 2010]. Disponível: http://www.opas.org.br/informacao/ UploadArq/LIVRO_Informe_de_Situação_WEB.pdf

9. Ministério da Saúde (BR). DATASUS. Estatísticas vitaisMortalidade e Nascidos Vivos. [Internet] 2008. [acesso em 20 mar 2010]. Disponível: http://tabnet.datasus.gov.br/cgi/ idb2006/matriz.htm\#mort

10. Ministério da Saúde (BR). DATASUS. Estatísticas vitaisMortalidade e Nascidos Vivos. [Internet] 2009. [acesso 30 jun 2010]. Disponível: http://www2.datasus.gov.br/DATASUS/ index.php?area $=0205$

11. Giglio MRP,Lamounier JA, Neto OLM, CésarCC. Baixo peso ao nascer em coorte de recém-nascidos em Goiânia-Brasil no ano de 2000. Rev bras ginecol obstet 2005; 27(3):130-6.
12. Duarte JLMB, Mendonça GAS. Fatores associados à morte neonatal em recém-nascidos de muito baixo peso em quatro maternidades no Município do Rio de Janeiro, Brasil. Cad. saude publica. 2005;21(1):181-91.

13. Ribeiro AM, Guimarães MJ, Lima MC, Sarinho SW, Coutinho SB. Fatores de risco para mortalidade neonatal em crianças com baixo peso ao nascer. Rev saude publica. 2009;43(2):246-55.

14. Freddi NA, Filho JOP, Fiori HH. Terapia com surfactante pulmonar exógeno em pediatria. J. pediatr. 2003;79(Supl.2):205-12.

15. Lansky S, França E, Leal MC. Mortalidade perinatal e evitabilidade: revisão da literatura. Rev saude publica. 2002;36(6):759-72.

16. Camargo ABM. A natimortalidade e a mortalidade perinatal em São Paulo. São Paulo em Perspec. 2008;22(1):30-47.

17. Ministério da Saúde (BR). Manual Técnico- Pré-natal e Puerpério: atenção qualificada e humanizada. Brasília. 2006.

18. Ministério da Saúde(BR). Painel de indicadores do SUS n ${ }^{\circ} 2$ Temático saúde da mulher. Brasília. [Internet] 1(2). [acesso 30 jun 2010]. Disponível: http://www.fiocruz.br/redeblh/media/ painelmulher.pdf

19. Puccini RF, Pedroso GC, Silva EMK, Araújo NS, Silva NN. Eqüidade na atenção pré-natal e ao parto em área da Região Metropolitana de São Paulo, 1996. Cad. saude publica. 2003;19(1):35-45. 